\title{
Comprehensive Involutive Systems
}

\author{
Vladimir Gerdt ${ }^{1}$ and Amir Hashemi ${ }^{2}$ \\ 1 Laboratory of Information Technologies, Joint Institute for Nuclear Research \\ 141980 Dubna, Russia, e-mail: gerdt@jinr.ru \\ 2 Department of Mathematical Sciences, Isfahan University of Technology \\ Isfahan, 84156-83111, Iran, e-mail: Amir.Hashemi@cc.iut.ac.ir
}

\begin{abstract}
In this paper we consider parametric ideals and introduce a notion of comprehensive involutive system. This notion plays the same role in theory of involutive bases as the notion of comprehensive Gröbner system in theory of Gröbner bases. Given a parametric ideal, the space of parameters is decomposed into a finite set of cells. Each cell yields the corresponding involutive basis of the ideal for the values of parameters in that cell. Using the Gerdt-Blinkov algorithm described in [6] for computing involutive bases and also the Montes DisPGB algorithm for computing comprehensive Gröbner systems [13], we present an algorithm for construction of comprehensive involutive systems. The proposed algorithm has been implemented in Maple, and we provide an illustrative example showing the stepby-step construction of comprehensive involutive system by our algorithm.
\end{abstract}

\section{Introduction}

One of the most important algorithmic objects in computational algebraic geometry is Gröbner basis. The notion of Gröbner basis was introduced and an algorithm for its construction was designed in 1965 by Buchberger in his Ph.D. thesis [3]. Later on, he discovered [4] two criteria for detecting some useless reductions that made the Gröbner bases method a practical tool to solve a wide class of problems in polynomial ideal theory and in many other research areas of science and engineering [5]. We refer to the monograph [2] for details on the theory of Gröbner bases.

The concept of comprehensive Gröbner bases can be considered as an extension of these bases for polynomials over fields to polynomials with parametric coefficients. This extension plays an important role in application to constructive algebraic geometry, robotics, electrical network, automatic theorem proving and so on (see, for example, [11-14]). Comprehensive Gröbner bases and equivalent to them comprehensive Gröbner systems were introduced in 1992 by Weispfenning [22]. He proved that any parametric polynomial ideal has a comprehensive Gröbner basis and described an algorithm to compute it. In 2002, Montes [13] proposed a more efficient algorithm (DisPGB) for computing comprehensive Gröbner systems. A year later Weispfenning in [21] proved the existence of a canonical comprehensive Gröbner basis. In 2003, Sato and Suzuki [17] introduced the concept of alternative comprehensive Gröbner bases. Then in 2006, Manubens and Montes in [11] by using discriminant ideal improved DisPGB, and in [12] they introduced an algorithm for computing minimal canonical Gröbner systems. Also in 2006, Sato and Suzuki [18] (see also [19]) suggested an important computational improvement for comprehensive Gröbner bases by constructing the reduced Gröbner bases in polynomial rings over ground fields. In 2010, Kapur, Sun and Wang [10], by combining Weispfenning's algorithm [22] with Suzuki and Sato's algorithm [18], proposed a new algorithm for computing comprehensive Gröbner systems. More recently, in 2010, Montes and Wibmer in [15] presented the GRÖBNERCOVER algorithm (its implementation in Singular is available at http://www-ma2.upc.edu/ montes/) which computes a finite partition of the parameter space into locally closed subsets together with polynomial data and such that the reduced Gröbner basis for given values of parameters can immediately be determined from the partition.

Involutive bases form an important class of Gröbner bases. The theory of involutive bases goes back to the seminal works of French mathematician Janet. In the 20s of the last century, he developed [9] a constructive approach to analysis of certain systems of partial differential equations 
based on their completion to involution (cf. [20]). Inspired by the involution methods described in the book by Pommaret [16], Zharkov and Blinkov [23] introduced the concept of involutive polynomial bases in commutative algebra in the full analogy with the concept of involutive systems of homogeneous linear partial differential equations with constant coefficients and in one dependent variable. Besides, Zharkov and Blinkov designed the first algorithm for construction of involutive polynomial bases. The particular form of an involutive basis they used is nowadays called Pommaret basis [20].

Gerdt and Blinkov [7] proposed a more general concept of involutive bases for polynomial ideals and designed efficient algorithmic methods to construct such bases. The underlying idea of the involutive approach is to translate the methods originating from Janet's approach into the polynomial ideals theory in order to provide a method for construction of involutive bases by combining algorithmic ideas in the theory of Gröbner bases with constructive ideas in the theory of involutive differential systems. In doing so, Gerdt and Blinkov [7] introduced the concept of involutive division. Moreover, they derived the involutive form of Buchberger's criteria. This led to a strong computational tool which is a serious alternative to the conventional Buchberger algorithm. We refer to Seiler's book [20] for a comprehensive study and application of involution to commutative algebra and geometric theory of partial differential equations.

In this paper, we introduce a notion of comprehensive involutive systems. For a parametric ideal, we decompose the space of parameters into a finite set of cells, and for each cell we yield the corresponding involutive basis of the ideal. Thereby, for each values of parameters, we find first a cell containing these values. Then, by substituting these values into the corresponding basis, we get the involutive basis of the given ideal. Based on the Gerdt-Blinkov involutive (abbreviated below by GBI) algorithm as described in [6] and also the Montes DisPGB algorithm [13], we present an algorithm for constructing comprehensive involutive systems. The proposed algorithm has been implemented in Maple, and we provide an illustrative example showing the step-by-step results of the algorithm.

The paper is structured as follows. Section 2 contains the basic definitions and notations related to comprehensive Gröbner systems, and a short description of the DisPGB algorithm. The basic definitions and notations from the theory of involutive bases are given in Section 3. In Section 4, the notion of comprehensive involutive system is introduced, and an algorithm for construction of such systems is described. In Section 5, we give an example illustrating in detail the performance of the algorithm of Section 4.

\section{Comprehensive Gröbner Systems}

In this section, we recall the basic definitions and notations in theory of comprehensive Gröbner systems and briefly describe the DisPGB algorithm.

Let $R=K[\mathbf{x}]$ be a polynomial ring, where $\mathbf{x}=x_{1}, \ldots, x_{n}$ is a sequence of variables and $K$ is an arbitrary field. Below, we denote a monomial $x_{1}^{\alpha_{1}} \cdots x_{n}^{\alpha_{n}} \in R$ by $\mathbf{x}^{\alpha}$ where $\alpha=\left(\alpha_{1}, \ldots, \alpha_{n}\right) \in \mathbb{N}^{n}$ is a sequence of non-negative integers. We shall use the notations $\operatorname{deg}_{i}\left(\mathbf{x}^{\alpha}\right):=\alpha_{i}, \operatorname{deg}\left(\mathbf{x}^{\alpha}\right):=$ $\sum_{i=1}^{n} \alpha_{i}$. An admissible monomial ordering on $R$ is a total order $\prec$ on the set of all monomials such that for any $\alpha, \beta, \gamma \in \mathbb{N}^{n}$ the following holds:

$$
\mathbf{x}^{\alpha} \succ \mathbf{x}^{\beta} \Longrightarrow \mathbf{x}^{\alpha+\gamma} \succ \mathbf{x}^{\beta+\gamma}, \quad \mathbf{x}^{\alpha} \neq 1 \Longrightarrow \mathbf{x}^{\alpha} \succ 1 .
$$

A typical example of admissible monomial ordering is the lexicographical ordering, denoted by $\prec_{\text {lex }}$. If $\mathbf{x}^{\alpha}, \mathbf{x}^{\beta} \in R$ are two monomials, then $\mathbf{x}^{\alpha} \prec_{\text {lex }} \mathbf{x}^{\beta}$ if the leftmost nonzero entry of $\beta-\alpha$ is positive. Another typical example is the degree-reverse-lexicographical ordering denoted by $\prec_{\text {degrevlex }}$ and defined as $\mathbf{x}^{\alpha} \prec_{\text {degrevlex }} \mathbf{x}^{\beta}$ if $\operatorname{deg}\left(\mathbf{x}^{\alpha}\right)>\operatorname{deg}\left(\mathbf{x}^{\beta}\right)$ or $\operatorname{deg}\left(\mathbf{x}^{\alpha}\right)=\operatorname{deg}\left(\mathbf{x}^{\beta}\right)$ and the rightmost nonzero entry of $\beta-\alpha$ is negative.

We shall write $I=\left\langle f_{1}, \ldots, f_{k}\right\rangle$ for the ideal $I$ in $R$ generated by the polynomials $f_{1}, \ldots, f_{k} \in$ $R$. Let $f \in R$ and $\prec$ be a monomial ordering on $R$. The leading monomial of $f$ is the largest monomial (with respect to $\prec$ ) occurring in $f$, and we denote it by $\operatorname{LM}(f)$. If $F \subset R$ is a set of polynomials, then we denote by $\operatorname{LM}(F)$ the set $\{\operatorname{LM}(f) \mid f \in F\}$ of its leading monomials. The 
leading coefficient of $f$, denoted by $\operatorname{LC}(f)$, is the coefficient of $\operatorname{LM}(f)$. The leading term of $f$ is $\mathrm{LT}(f)=\mathrm{LC}(f) \operatorname{LM}(f)$. The leading term ideal of $I$ is defined as $\operatorname{LT}(I)=\langle\operatorname{LT}(f) \mid f \in I\rangle$.

A finite set $G=\left\{g_{1}, \ldots, g_{k}\right\} \subset I$ is called a Gröbner basis of $I$ if $\operatorname{LT}(I)=\left\langle\operatorname{LT}\left(g_{1}\right), \ldots, \operatorname{LT}\left(g_{k}\right)\right\rangle$. For more details and definitions related to Gröbner bases we refer to [2].

Now consider $F=\left\{f_{1}, \ldots, f_{k}\right\} \subset S:=K[\mathbf{a}, \mathbf{x}]$ where $\mathbf{a}=a_{1}, \ldots, a_{m}$ is a sequence of parameters. Let $\prec_{\mathbf{x}}\left(\right.$ resp. $\left.\prec_{\mathbf{a}}\right)$ be a monomial ordering for the power products of $x_{i}$ 's (resp. $a_{i}$ 's). We also need a compatible elimination product ordering $\prec_{\mathbf{x}, \mathbf{a}}$. It is defined as follows: For all $\alpha, \gamma \in \mathbb{Z}_{\geq 0}^{n}$ and $\beta, \delta \in \mathbb{Z}_{\geq 0}^{m}$

$$
\mathbf{x}^{\gamma} \mathbf{a}^{\delta} \prec_{\mathbf{x}, \mathbf{a}} \mathbf{x}^{\alpha} \mathbf{a}^{\beta} \Longleftrightarrow \mathbf{x}^{\gamma} \prec_{\mathbf{x}} \mathbf{x}^{\alpha} \text { or } \mathbf{x}^{\gamma}=\mathbf{x}^{\alpha} \text { and } \mathbf{a}^{\delta} \prec_{\mathbf{a}} \mathbf{a}^{\beta}
$$

Now, we recall the definition of a comprehensive Gröbner system for a parametric ideal.

Definition 1. ([22]) A triple set $\left\{\left(G_{i}, N_{i}, W_{i}\right)\right\}_{i=1}^{\ell}$ is called a comprehensive Gröbner system for $\langle F\rangle$ w.r.t $\prec_{\mathbf{x}, \mathbf{a}}$ if for any $i$ and any homomorphism $\sigma: K[\mathbf{a}] \rightarrow K^{\prime}$ (where $K^{\prime}$ is a field extension of $K$ ) satisfying

$$
\text { (i) }\left(\forall p \in N_{i} \subset K[\mathbf{a}]\right)[\sigma(p)=0], \quad(i i)\left(\forall q \in W_{i} \subset K[\mathbf{a}]\right)[\sigma(q) \neq 0]
$$

we have $\sigma\left(G_{i}\right)$ is a Gröbner basis for $\sigma(\langle F\rangle) \subset K^{\prime}[\mathbf{x}]$ w.r.t. $\prec_{\mathbf{x}}$.

For simplification, we shall use the abbreviation CGS to refer to a comprehensive Gröbner system, and CGSs in the plural case. For each $i$, the set $N_{i}$ (resp. $W_{i}$ ) is called a (resp. non-) null conditions set. The pair $\left(N_{i}, W_{i}\right)$ is called a specification of the homomorphism $\sigma$ if both conditions in the above definition are satisfied.

Example 1. Let $F=\left\{a x^{2} y-y^{3}, b x+y^{2}\right\} \subset K[a, b, x, y]$ where $\mathbf{a}=a, b$ and $\mathbf{x}=x, y$. Let us consider the lexicographical monomial ordering $b \prec_{\text {lex }} a$ on the parameters and on the variables $y \prec_{\operatorname{lex}} x$ as well. Using the DisPGB algorithm we can compute a CGS for $\langle F\rangle$ which is equal to

$$
\begin{array}{llc}
\left\{-b^{2} y^{3}+a y^{5}, b x+y^{2}\right\} & \{\} & \{a, b\} \\
\left\{x^{2} y, y^{2}\right\} & \{b\} & \{a\} \\
\left\{y^{3}, b x+y^{2}\right\} & \{a\} & \{b\} \\
\left\{y^{2}\right\} & \{a, b\} & \{\} .
\end{array}
$$

For instance, if $a=0, b=2$, then the third element of this system corresponds to this specialization. Therefore, $\left\{y^{3}, 2 x+y^{2}\right\}$ is a Gröbner basis for the ideal $\left.\langle F\rangle\right|_{a=0, b=2}=\left\langle-y^{3}, 2 x+y^{2}\right\rangle$.

Remark that, by the above definition, a CGS is not unique for a given parametric ideal, and one can find other partitions for the space of parameters, and, therefore, other CGSs for the parametric ideal.

Now, we briefly describe the Montes DisPGB algorithm to compute CGSs for parametric ideals (see $[13,11]$ ). The main idea of DisPGB is based on discussing the nullity or not w.r.t. a given specification $(N, W)$ for the leading coefficients of the polynomials appearing at each step (this process is performed by the NewConD subalgorithm). Let us consider a set $F \subset S$ of parametric polynomials. Given a polynomial $f \in F$ and a specification $(N, W)$, NEwCond is called. Three cases are possible: If $\mathrm{LC}(f)$ specializes to zero w.r.t. $(N, W)$, we replace $f$ by $f-\mathrm{LT}(f)$, and then start again. If $\mathrm{LC}(f)$ specializes to a nonzero element we continue with the next polynomial in $F$. Otherwise (if $\operatorname{LC}(f)$ is not decidable, i.e. we can't decide whether or not it is null w.r.t. $(N, W)$ ), the subalgorithm BRANCH is called to create two complementary cases by assuming $\operatorname{LC}(f)=0$ and $\operatorname{LC}(f) \neq 0$. Therefore, two new disjoint branches with the specifications $(N \cup\{\operatorname{LC}(f)\}, W)$ and $(N, W \cup\{\mathrm{LC}(f)\})$ are made. This procedure is continued until every polynomial in $F$ has a nonnull leading coefficient w.r.t. the current specification. Then, we proceed with CondPGB: This algorithm receives, as an input, a set of parametric polynomials and a specification $(N, W)$ and, by applying Buchberger's algorithm, creates new polynomials. When a new polynomial is generated, NEWCOND verifies whether its leading coefficient leads to a new condition or not. If a new condition is found, then the subalgorithm stops, and BRANCH is called to make two new disjoint branches. Otherwise, the process is continued and computes a Gröbner basis for $\langle F\rangle$, according to the current specification. The collection of these bases, together with the corresponding specifications yields a CGS for $\langle F\rangle$. 


\section{Involutive Bases}

Now we recall the basic definitions and notations concerning involutive bases and present below the general definition of involutive bases. First of all, we describe the cornerstone notion of involutive division [7] as a restricted monomial division [6] which, together with a monomial ordering, determines properties of an involutive basis. This makes the main difference between involutive bases and Gröbner bases. The idea behind involutive division is to partition the variables into two subsets of multiplicative and nonmultiplicative variables, and only the multiplicative variables can be used in the divisibility relation.

Definition 2. [7,6] An involutive division $\mathcal{L}$ on the set of monomials of $R$ is given, if for any finite set $U$ of monomials and any $u \in U$, the set of variables is partitioned into subsets $M_{\mathcal{L}}(u, U)$ of multiplicative and $N M_{\mathcal{L}}(u, U)$ of nonmultiplicative variables such that

1. $u, v \in U, u \mathcal{L}(u, U) \cap v \mathcal{L}(v, U) \neq \emptyset \Longrightarrow u \in v \mathcal{L}(v, U)$ or $v \in u \mathcal{L}(u, U)$,

2. $v \in U, v \in u \mathcal{L}(u, U) \Longrightarrow \mathcal{L}(v, U) \subset \mathcal{L}(u, U)$,

3. $u \in V$ and $V \subset U \Longrightarrow \mathcal{L}(u, U) \subset \mathcal{L}(u, V)$,

where $\mathcal{L}(u, U)$ denotes the set of all monomials in the variables in $M_{\mathcal{L}}(u, U)$. If $v \in u \mathcal{L}(u, U)$, then we call $u$ an $\mathcal{L}$-(involutive) divisor of $v$, and we write $\left.u\right|_{\mathcal{L}} v$. If $v$ has no involutive divisor in a set $U$, then it is $\mathcal{L}$-irreducible modulo $U$.

In this paper, we are concerned with the wide class [8] of involutive divisions determined by a permutation $\rho$ on the indices of variables and by a total monomial ordering $\sqsupset$ which is either admissible or the inverse of an admissible ordering. This class is defined by

$$
(\forall u \in U) \quad\left[N M_{\sqsupset}(u, U)=\bigcup_{v \in U \backslash\{u\}} N M_{\sqsupset}(u,\{u, v\})\right]
$$

where

$$
N M_{\sqsupset}(u,\{u, v\}):=\left\{\begin{array}{l}
\text { if } u \sqsupset v \text { or }(u \sqsubset v \wedge v \mid u) \text { then } \emptyset \\
\text { else }\left\{x_{\rho(i)}\right\}, i=\min \left\{j \mid \operatorname{deg}_{\rho(j)}(u)<\operatorname{deg}_{\rho(j)}(v)\right\} .
\end{array}\right.
$$

Remark 1. The involutive Janet division introduced and studied in [7] is generated by formulae (1)-(2) if $\sqsupset$ is the lexicographic monomial ordering $\succ_{\text {lex }}$ and $\rho$ is the identical permutation. The partition of variables used by Janet himself [9] (see also [20]) is generated by $\succ_{\text {lex }}$ as well with the permutation which is inverse to the identical one:

$$
\rho=\left(\begin{array}{cccc}
1 & 2 & \ldots & n \\
n & n-1 & \ldots & 1
\end{array}\right)
$$

Throughout this paper $\mathcal{L}$ is assumed to be a division of the class (1)-(2). Now, we define an involutive basis.

Definition 3. Let $I \subset R$ be an ideal, $\prec$ be a monomial ordering on $R$ and $\mathcal{L}$ be an involutive division. A finite set $G \subset I$ is an involutive basis of $I$ if for all $f \in I$ there exists $g \in G$ such that $\left.\mathrm{LM}(g)\right|_{\mathcal{L}} \mathrm{LM}(f)$. An involutive basis $G$ is minimal if for any other involutive basis $\tilde{G}$ the inclusion $\operatorname{LM}(G) \subseteq \operatorname{LM}(\tilde{G})$ holds.

¿From this definition and from that for Gröbner basis [3,2] it follows that an involutive basis of an ideal is its Gröbner basis, but the converse is not always true.

Remark 2. By using an involutive division in the division algorithm for polynomial rings, we obtain an involutive division algorithm. If $G$ is an involutive basis for an involutive division $\mathcal{L}$, we use $\mathrm{NF}_{\mathcal{L}}(f, G)$ to denote $\mathcal{L}$-normal form of $f$ modulo $G$, i.e. the remainder of $f$ on the involutive division by $G$. A polynomial set $F$ is $\mathcal{L}$-autoreduced if $f=\mathrm{NF}_{\mathcal{L}}(f, F \backslash\{f\})$ for every $f \in F$. 
The following theorem provides an algorithmic characterization of involutive bases which is an involutive analogue of the Buchberger characterization of Gröbner bases.

Theorem 1. ([7,8]) Given an ideal $I \subset R$, an admissible monomial ordering $\prec$ on $R$ and an involutive division $\mathcal{L}$, a finite subset $G \subset I$ is an involutive basis of $I$ if for each $f \in G$ and each $x \in N M_{\mathcal{L}}(\operatorname{LM}(f), \operatorname{LM}(G))$ the equality $\mathrm{NF}_{\mathcal{L}}(x f, G)=0$ holds. An involutive basis exists for any $I, \mathcal{L}$ and $\prec . A$ monic and $\mathcal{L}$-autoreduced involutive basis is uniquely defined by $I$ and $\prec$.

\section{Comprehensive Involutive Systems}

In this section, like the concept of comprehensive Gröbner systems, we define the new notion of comprehensive involutive system for a parametric ideal. Then, based on the GBI algorithm [6] and the Montes DisPGB algorithm [13], we propose an algorithm for computing comprehensive involutive systems.

Definition 4. Consider a finite set of parametric polynomials $F \subset S=K[\mathbf{a}, \mathbf{x}]$ where $K$ is a field, $\mathbf{x}=x_{1}, \ldots, x_{n}$ is a sequence of variables and $\mathbf{a}=a_{1}, \ldots, a_{m}$ is a sequence of parameters, $\prec_{\mathbf{x}}$ (resp. $\prec_{\mathbf{a}}$ ) is a monomial ordering involving the $x_{i}$ 's (resp. $a_{i}$ 's), and $\mathcal{L}$ is an involutive division on $K[\mathbf{x}]$. Let $M=\left\{\left(G_{i}, N_{i}, W_{i}\right)\right\}_{i=1}^{\ell}$ be a finite triple set where sets $N_{i}, W_{i} \subset K[\mathbf{a}]$ and $G_{i} \subset S$ are finite. The set $M$ is called an $(\mathcal{L}-)$ comprehensive involutive system for $\langle F\rangle$ w.r.t $\prec_{\mathbf{x}, \mathbf{a}}$ if for each $i$ and for each homomorphism $\sigma: K[a] \rightarrow K^{\prime}$ (where $K^{\prime}$ is a field extension of $K$ ) satisfying

$$
\text { (i) }\left(\forall p \in N_{i}\right)[\sigma(p)=0], \quad(i i)\left(\forall q \in W_{i}\right)[\sigma(q) \neq 0]
$$

$\sigma\left(G_{i}\right)$ is an $(\mathcal{L}-)$ involutive basis for $\sigma(\langle F\rangle) \subset K^{\prime}[\mathbf{x}]$. We use the abbreviation CIS (resp. CISs) to stand for comprehensive involutive system (resp. systems). $M$ is called minimal, if for each $i$, the set $\sigma\left(G_{i}\right)$ is a minimal involutive basis.

Given a CGS, one can straightforwardly compute a CIS by using the following proposition.

Proposition 1. Let $G=\left\{g_{1}, \ldots, g_{k}\right\}$ be a minimal Gröbner basis of an ideal $I \subset K\left[x_{1}, \ldots, x_{n}\right]$ for a monomial ordering $\prec$. Let $h_{i}=\max _{g \in G}\left\{\operatorname{deg}_{i}(\operatorname{LM}(g))\right\}$. Then the set of products

$$
\left\{m g \mid g \in G, m \text { is a monomial s.t. }(\forall i)\left[\operatorname{deg}_{i}(m) \leq h_{i}-\operatorname{deg}_{i}(\operatorname{LM}(g))\right]\right\}
$$

is an $\mathcal{L}$-involutive basis of $I$.

Proof. Denote $\operatorname{LM}(G)$ by $U$. From (1)-(2) it follows

$$
\left.(\forall u \in U)\left(\forall x_{i} \in N M_{\mathcal{L}}(u, U)\right)\right)\left[\operatorname{deg}_{i}(u)<h_{i}\right] .
$$

It is also clear that if we enlarge $G$ with a (not necessarily nonmultiplicative) prolongation $g x_{j}$ of its element $g \in G$ such that $\operatorname{deg}_{j}(\operatorname{LM}(g))<h_{j}$, then (4) holds for the enlarged leading monomial set $U:=U \cup\left\{\operatorname{LM}(g) x_{j}\right\}$ as well. Consider completion $\bar{G}$ of the polynomial set $G$ with all possible prolongations of its elements satisfying (3) and denote the monomial set $\operatorname{LM}(\bar{G})$ by $\bar{U}$. Then

$$
(\forall u \in \bar{U})\left(\forall x \in N M_{\mathcal{L}}(u, U)\right)(\exists v \in \bar{U})\left[\left.v\right|_{\mathcal{L}} u x\right] .
$$

This means, by Theorem 1 , that the monomial set $\bar{U}$ is an involutive basis of $\langle\operatorname{LM}(G)\rangle$. Now, since $G$ is a Gröbner basis of $I$ we have $\operatorname{LT}(I)=\langle\operatorname{LM}(G)\rangle$, and hence $\operatorname{LT}(I)=\langle\operatorname{LM}(\bar{G})\rangle$. Therefore, $\bar{G}$ is an involutive basis of $I$ by Definition 3 .

Example 2. Let $F=\left\{a x^{2}, b y^{2}\right\} \subset \mathbb{K}[\mathbf{a}, \mathbf{x}]$ where $\mathbf{a}=a, b$ and $\mathbf{x}=x, y$. Let also $b \prec_{\text {lex }} a$ and $y \prec_{\text {lex }} x$. Then, $F$ is a CGS for any sets of null and nonnull conditions. Using Proposition 1, we can construct the following Janet basis of $\langle F\rangle$ which is a GIS for any sets of null and nonnull conditions:

$$
\left\{a x^{2}, b y^{2}, a y x^{2}, a y^{2} x^{2}, b x y^{2}, b x^{2} y^{2}\right\} .
$$


On the other hand, the algorithm that we present below computes the following minimal Janet CIS for $\langle F\rangle$ :

$\begin{array}{lll}\left\{a x^{2}, b y^{2}, b x y^{2}\right\} & \{\} & \{a, b\} \\ \left\{a x^{2}\right\} & \{b\} & \{a\} \\ \left\{b y^{2}\right\} & \{a\} & \{b\} \\ \{0\} & \{a, b\} & \{\} .\end{array}$

Remark 3. Using Proposition 1, we cannot directly compute a minimal CIS from a given CGS. Indeed, to do this, we must examine the leading coefficients of each Gröbner basis in the CGS, and this may lead to further partitions of the space of parameters. Moreover, the CIS computed by this way may be too large, since many prolongations constructed by means of (3) may be useless. That is why, based on the GBI algorithm [6] and on the Montes DisPGB algorithm [13], we propose a more efficient algorithm for computing minimal CISs.

Now we describe the structure of polynomials that is used in our new algorithm. To avoid unnecessary reductions (during the computation of involutive bases) by applying the involutive form of Buchberger's criteria (see [6]), we need to supply polynomials with additional structural information.

Definition 5. [6] An ancestor of a polynomial $f \in F \subset R \backslash\{0\}$, denoted by anc $(f)$, is a polynomial $g \in F$ of the smallest $\operatorname{deg}(\operatorname{LM}(g))$ among those satisfying $\operatorname{LM}(f)=u \operatorname{LM}(g)$ where $u$ is either the unit monomial or a power product of nonmultiplicative variables for $\operatorname{LM}(g)$ and such that $\mathrm{NF}_{\mathcal{L}}(f-u g, F \backslash\{f\})=0$ if $f \neq u g$.

Below we show how to use this concept to apply the involutive form of Buchberger's criteria. In what follows, we store each polynomial $f$ as the $p=[f, g, V]$ where $f=\operatorname{poly}(p)$ is the polynomial part of $p, g=\operatorname{anc}(p)$ is the ancestor of $f$ and $V=N M(p)$ is the list of nonmultiplicative variables of $f$ have been already used to construct prolongations of $f$ (see the for-loop 20-23 in the subalgorithm GBI). If $P$ is a set of triples, we denote by $\operatorname{poly}(P)$ the set $\{\operatorname{poly}(p) \mid p \in P\}$. If no confusion arises, we may refer to a triple $p$ instead of poly $(p)$, and vice versa.

We present now the main algorithm CoMINvSYs which computes a minimal CIS for a given ideal. It should be noted that we use the subalgorithms NEwCOND and CANSPEC (resp. TAILNormalForm) as they have (resp. it has) been presented in [13] (resp. [6]), and recall them for the sake of completeness. Also, we use the subalgorithm Branch (resp. GBI, HeadReduce and HEADNORMALFORM) from [13] (resp. [6]) with some appropriate modifications.

\section{Algorithm ComInvSys}

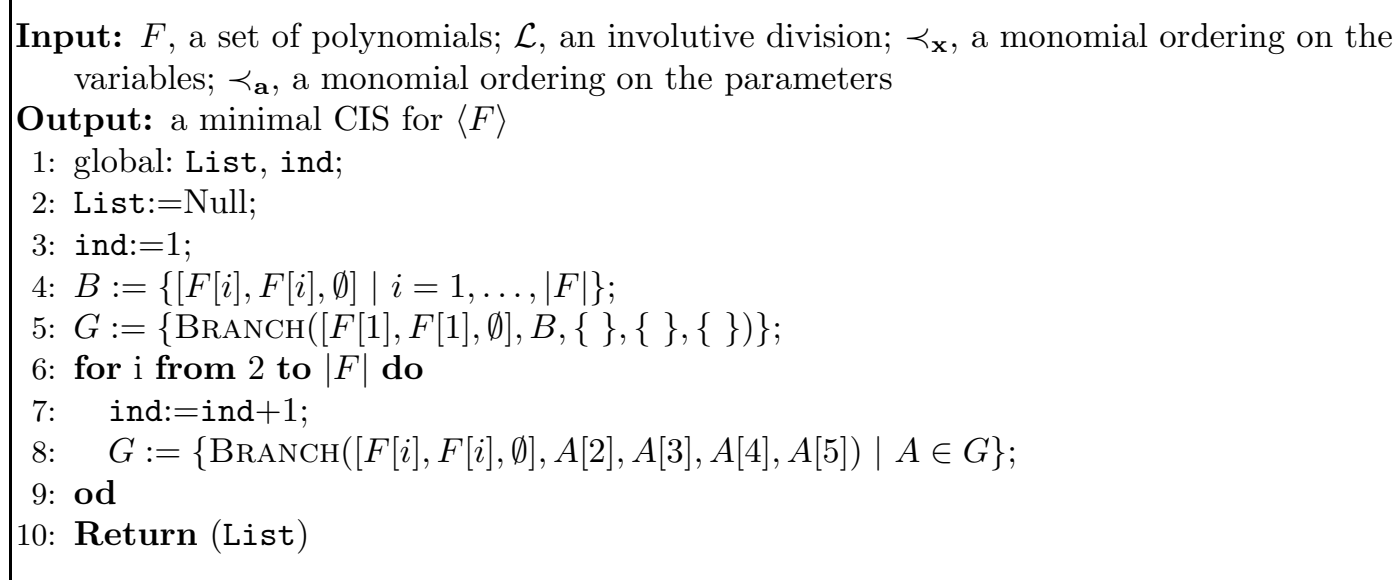

In the above algorithm, List is a global variable to which we add any computed involutive basis together with its corresponding specification to form the final CIS. That is why, at the beginning of computation we must set it to the empty list (see BRANCH). Note that here and in BRANCH, we 
use $|F|$ to denote the number of polynomials in the input set $F$. The variable ind is also a global variable, and we use it to examine all the leading coefficients of the elements in $F$ (see BRANCH). Once we are sure about the non-nullity of these coefficients, then we start the involutive basis computation. Indeed, BRANCH inputs a triple $p=[f, g, V]$, a set $B$ of examined and processed polynomials, a set $N$ of null conditions, a set $W$ of nonnull conditions and a set $P$ of non-processed polynomials. Then, it analyses the leading coefficient of $f$ w.r.t. $N$ and $W$. Now, two cases are possible:

- ind $<|F|$ : If $\mathrm{LC}(f)$ is not decidable by $N$ and $W$ then we create two complementary cases by assuming $\mathrm{LC}(f)=0$ and $\mathrm{LC}(f) \neq 0$. Then we pass to the next polynomial in $F$.

- ind $=|F|$ : We are now sure that we have examined all the leading coefficients of the elements in $F$ (except possibly the very last one which is to be $f$ ). If $\mathrm{LC}(f)$ is not decidable by $N$ and $W$ then we again create two complementary cases with $\mathrm{LC}(f)=0$ and $\mathrm{LC}(f) \neq 0$. Otherwise, we continue to process the polynomials in $P$ by using the GBI algorithm. If $P=\emptyset$ this means that $B$ is an involutive basis consistent with the conditions in $N$ and $W$, and we add $(B, N, W)$ to List.

It is worth noting that if the input specification of BRANCH is incompatible, then it stops the process only for the corresponding branch, and continues the construction of other branches. Moreover, using the above notations, if ind $<|F|$ and no new condition is detected, then BRANCH returns an element of the form $\left(p, \bar{B}^{N^{\prime}}, N^{\prime}, W^{\prime}, P\right)$ where $p$ is a triple, $N^{\prime}, W^{\prime}$ are two sets of conditions, $\bar{B}^{N^{\prime}}$ is the normal form of a specializing basis $B$ and $P$ is a set of non-examined triples. Otherwise, it calls itself to create the new branches. Finally, if ind $=|F|$, then the algorithm does not return anything and completes the global variable List.

\section{Subalgorithm BRANCH}

Input: $p$, a triple; $B$, a specializing basis; $N$, a set of null conditions; $W$, a set of nonnull conditions; $P$, a set of non-examined triples

Output: It stores the refined $\left(B^{\prime}, N^{\prime}, W^{\prime}, P^{\prime}\right)$, and creates two new vertices when necessary or marks the vertex as terminal

1: $p=[f, g, V]$;

2: $($ test $, N, W):=\operatorname{CANSpeC}(N, W)$;

3: if test=false then

4: Return STOP (incompatible specification has been detected)

5: $\mathbf{f i}$

6: $\left(c d, f^{\prime}, N^{\prime}, W^{\prime}\right):=\operatorname{NewCOND}(f, N, W)$;

7: $p:=\left[f^{\prime}, \bar{g}^{N^{\prime}}, V\right]\left(\bar{g}^{N^{\prime}}\right.$ denotes the remainder of the division of $g$ by $\left.N^{\prime}\right)$;

8: if ind $<|F|$ and $c d \neq\{\}$ then

9: $\operatorname{Branch}\left(p, B, N^{\prime}, W^{\prime} \cup c d, P\right) ; \quad \operatorname{Branch}\left(p, B, N^{\prime} \cup c d, W^{\prime}, P\right)$;

10: $\mathrm{fi}$

11: if ind $<|F|$ and $c d=\{\}$ then

12: $\quad$ Return $\left(p, \bar{B}^{N^{\prime}}, N^{\prime}, W^{\prime}, P\right)$

13: $\mathbf{f i}$

14: if $c d=\{\}$ then

15: $\quad\left(\right.$ test $\left., p^{\prime}, B^{\prime}, N^{\prime}, W^{\prime}, P^{\prime}\right):=\mathrm{GBI}\left(B, N^{\prime}, W^{\prime}, P\right)$;

16: if test then

17: $\quad$ List: $=$ List,$\left(B^{\prime}, N^{\prime}, W^{\prime}\right)$;

18: $\quad$ else

19: $\operatorname{BRANCH}\left(p^{\prime}, B^{\prime}, N^{\prime}, W^{\prime}, P^{\prime}\right)$;

20: fi

21: else

22: $\operatorname{Branch}\left(p, B, N^{\prime}, W^{\prime} \cup c d, P\right) ; \quad \operatorname{Branch}\left(p, B, N^{\prime} \cup c d, W^{\prime}, P\right)$;

23: fi 
The subalgorithm CANSPEC produces a quasi-canonical representation for a given specification. Its subalgorithm FACVAR invoked in lines 1 and 13 returns the set of factors of its input polynomial.

Definition 6. ([13]) A specification $(N, W)$ is called quasi-canonical if

- $N$ is the reduced Gröbner basis w.r.t. $\prec \mathbf{a}$ of the ideal containing all polynomials that specialize to zero in $K[\mathbf{a}]$.

- The polynomials in $W$ specializing to non-zero are reduced modulo $N$ and irreducible over $K[\mathbf{a}]$

$-\prod_{q \in W} q \notin \sqrt{\langle N\rangle}$.

- The polynomials in $N$ are square-free over $K[\mathbf{a}]$.

- If some $p \in N$ is factorized, then no factor of $p$ belongs to $W$.

\section{Subalgorithm CANSPEC}

Input: $N$, a set of null conditions; $W$, a set of nonnull conditions

Output: true if $N$ and $W$ are compatible and false otherwise; $\left(N^{\prime}, W^{\prime}\right)$, a quasi-canonical representation of $(N, W)$

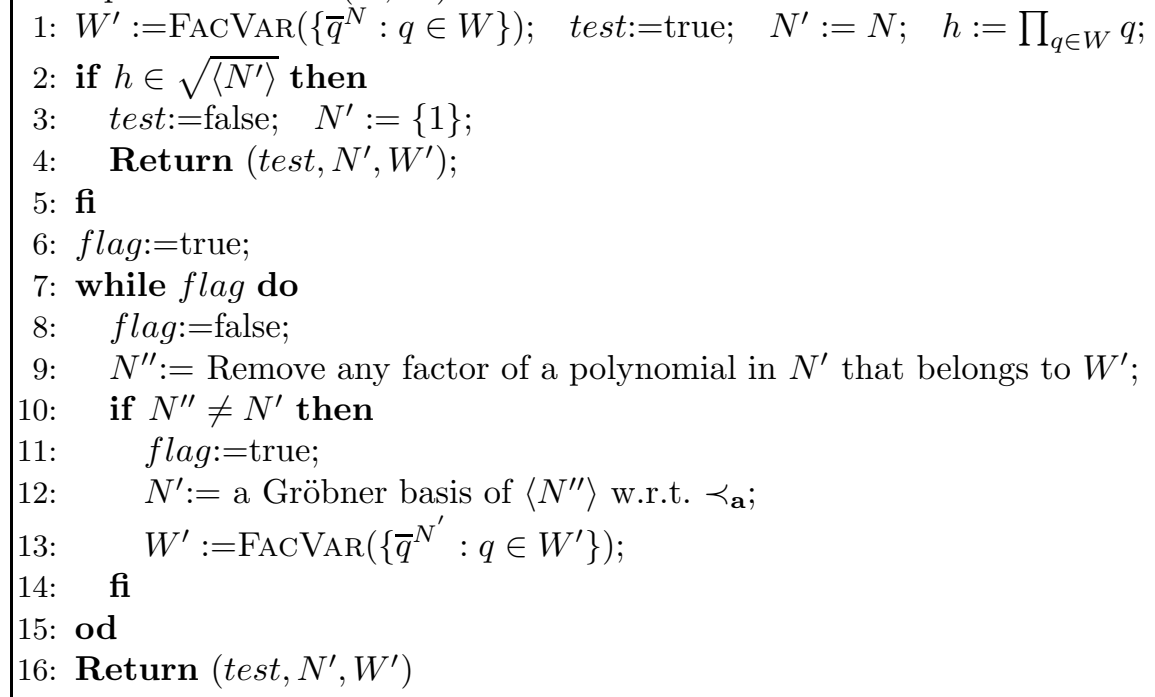

Subalgorithm NEwCOND

Input: $f$, a parametric polynomial; $N$, a set of null conditions; $W$, a set of nonnull conditions

Output: $c d$, a new condition; $f^{\prime}$, a parametric polynomial; $N^{\prime}$, a set of null conditions; $W^{\prime}$, a set of nonnull conditions

1: $f^{\prime}:=f ;$ test $:=$ true; $\quad N^{\prime}:=N ; \quad \mathrm{cd}:=\{\}$

2: while test do

3: if $\operatorname{LC}\left(f^{\prime}\right) \in \sqrt{\left\langle N^{\prime}\right\rangle}$ then

4: $\quad N^{\prime}:=$ a Gröbner basis for $\left\langle N^{\prime}, \operatorname{LC}\left(f^{\prime}\right)\right\rangle$ w.r.t. $\prec$ a

5: $\quad f^{\prime}:=f^{\prime}-\mathrm{LT}(f)$

6: $\quad$ else

7: $\quad$ test $:=$ false;

8: $\mathbf{f i}$

9: od

10: $f^{\prime}:={\overline{f^{\prime}}}^{N^{\prime}}$

11: $W^{\prime}:=\left\{\bar{w}^{N^{\prime}} \mid w \in W\right\}$;

12: $c d:=c d \cup$ FACVAR $\left(\operatorname{LC}\left(f^{\prime}\right)\right) \backslash W^{\prime}$;

13: $\operatorname{Return}\left(c d, f^{\prime}, N^{\prime}, W^{\prime}\right)$ 
We describe now the NewCond subalgorithm. When it is invoked in line 6 of BRANCH with the input data $(f, N, W)$, one of the two following cases may occur:

1. If $\operatorname{LC}(f)$ is decidable w.r.t. the specification $(N, W)$, then the subalgorithm returns:

(i) NewCond $(f-\operatorname{LT}(f), N, W)$ in the case when $\operatorname{LC}(f)$ specializes to zero w.r.t. $(N, W)$.

(ii) $(\emptyset, f, N, W)$ in the case when $\operatorname{LC}(f)$ does not specialize to zero w.r.t. $(N, W)$.

2. If $\operatorname{LC}(f)$ is not decidable w.r.t $(N, W)$, then NewCond returns $(c d, f, N, W)$ where set $c d$ contains one of the non-decidable factors (w.r.t $(N, W)$ ) of $\mathrm{LC}(f)$.

It should be emphasized that $\operatorname{FACVAR}\left(\operatorname{LC}\left(f^{\prime}\right)\right) \backslash W^{\prime}$ in line 12 returns only one factor of $\operatorname{LC}\left(f^{\prime}\right)$.

The subalgorithm GBI, presented below, is an extension of the algorithm InvolutiveBASIS

II described in [6]. The latter algorithm computes involutive bases and applies the involutive form of Buchberger's criteria to avoid some unnecessary reductions [7] (see also $[1,6]$ ). The criteria are applied in the subalgorithm HEadNormalForm (see line 7) that is invoked in line 5 of GBI.

Proposition 2. ([7,6]) Let $I \subset R$ be an ideal and $G \subset I$ be a finite set. Let also $\prec$ be a monomial ordering on $R$ and $\mathcal{L}$ be an involutive division. Then $G$ is an $\mathcal{L}$-involutive basis of $I$ if for all $f \in G$ and for all $x \in N M_{\mathcal{L}}(\operatorname{LM}(f), \operatorname{LM}(G))$ one of the two conditions holds:

1. $\mathrm{NF}_{\mathcal{L}}(x f, G)=0$.

2. There exists $g \in G$ with $\left.\operatorname{LM}(g)\right|_{\mathcal{L}} \operatorname{LM}(x f)$ satisfying one of the following conditions:

$\left(C_{1}\right) \operatorname{LM}(\operatorname{anc}(f)) \operatorname{LM}(\operatorname{anc}(g))=\operatorname{LM}(x f)$,

$\left(C_{2}\right) \operatorname{lcm}(\mathrm{LM}(\operatorname{anc}(f)), \operatorname{LM}(\operatorname{anc}(g)))$ is a proper divisor of $\operatorname{LM}(x f)$.

\section{Subalgorithm GBI}

Input: $B$, a specializing basis; $N$, a set of null conditions; $W$, set of nonnull conditions; $P$, set of non-examined triples

Output: If test=true, a minimal involutive basis for $\langle B\rangle$ w.r.t. $\mathcal{L}$ and $\prec_{\mathbf{x}, \mathbf{a}}$; otherwise, it returns a triple so that we must discuss the leading coefficient of its polynomial part

1: if $P=\emptyset$ then

2: $\quad$ Select $p \in B$ with no proper divisor of $\operatorname{LM}(\operatorname{poly}(p))$ in $\operatorname{LM}(\operatorname{poly}(B))$

3: $\quad T:=\{p\} ; \quad Q:=B \backslash\{p\} ;$

4: else

5: $\quad T:=B ; \quad Q:=P$

6: $\mathrm{fi}$

7: while $Q \neq \emptyset$ do

8: $\quad\left(\right.$ test $\left., p, T, N, W, Q^{\prime}\right):=\operatorname{HeadReduce}(T, N, W, Q)$;

9: $\quad$ if test $=$ false then

10: $\quad$ Return (false, $p, T, N, W, Q^{\prime}$ )

11: $\quad$ fi

12: $\quad Q:=Q^{\prime}$

13: $\quad$ Select and remove $p \in Q$ with no proper divisor of $\operatorname{LM}(\operatorname{poly}(p))$ in $\operatorname{LM}(\operatorname{poly}(Q))$;

14: $\quad$ if $\operatorname{poly}(p)=\operatorname{anc}(p)$ then

15: $\quad$ for $q \in T$ whose $\operatorname{LM}(\operatorname{poly}(q))$ is a proper multiple of $\operatorname{LM}(\operatorname{poly}(p))$ do

16: $\quad Q:=Q \cup\{q\} ; \quad T:=T \backslash\{q\} ;$

17: $\quad$ od

18: $\quad$ fi

19: $\quad h:=$ TailNormalForm $(p, T) ; \quad T:=T \cup\{\{h, \operatorname{anc}(p), N M(p)\}\}$;

20: $\quad$ for $q \in T$ and $x \in N M_{\mathcal{L}}(\operatorname{LM}(\operatorname{poly}(q)), \operatorname{LM}(\operatorname{poly}(T))) \backslash N M(q)$ do

21: $\quad Q:=Q \cup\{\{x \operatorname{poly}(q)$, $\operatorname{anc}(q), \emptyset\}\}$;

22: $\quad N M(q):=N M(q) \cap N M_{\mathcal{L}}(\operatorname{LM}(\operatorname{poly}(q)), \operatorname{LM}(\operatorname{poly}(T))) \cup\{x\}$;

23: od

24: od

25: Return (true, $0, T, N, W,\{\}$ ) 
This algorithm invokes three subalgorithms HeadReduce, TailNormalForm and HeadNorMALFORM that we present below. The subalgorithm HEADREDUCE performs the involutive head reduction of polynomials in the input set of triples modulo the input specializing basis. The subalgorithm TailNormalForm (resp. HeadNormalForm) invoked in line 19 of GBI (resp. in line 4 of HEADREDUCE) computes the involutive tail normal form (resp. the involutive head normal form) of the polynomial in the input triple modulo the input specializing basis.

In the subalgorithm HeadNormalForm, the Boolean expression Criteria $(p, g)$ is true if at leat one of the conditions $\left(C_{1}\right)$ or $\left(C_{2}\right)$ in Proposition 2 are satisfied for $p$ and $g$, false otherwise. We refer to [6] for more details on the algorithm GBI and on its subalgorithms.

\section{Subalgorithm HeADREDUCE}

Input: $B$, a specializing basis; $N$, a set of null conditions; $W$, a set of nonnull conditions; $P$ a set of non-examined triples

Output: If test=true, the $\mathcal{L}$-head reduced form of $P$ modulo $B$; otherwise, it returns a triple such that we must examine the leading coefficient of its polynomial part

1: $S:=P ; \quad Q:=\emptyset$

: while $S \neq \emptyset$ do

Select and remove $p \in S$;

(test $, h, B, N, W):=\operatorname{HeadNormalForm}(p, B, N, W)$;

if test $=$ false then

Return (false, $p, B, N, W, S \cup Q$ )

fi

if $h \neq 0$ then

if $\operatorname{LM}(\operatorname{poly}(p)) \neq \mathrm{LM}(h)$ then

$Q:=Q \cup\{\{h, h, \emptyset\}\} ;$

else

$Q:=Q \cup\{p\}$

fi

else

if $\operatorname{LM}(\operatorname{poly}(p))=\operatorname{LM}(\operatorname{anc}(p))$ then

fi

$S:=S \backslash\{q \in S \mid \operatorname{anc}(q)=\operatorname{poly}(p)\} ;$

$\mathrm{fi}$

19: od

20: Return (true, $0, B, N, W, Q$ )

Subalgorithm TAILNORMALFORM

Input: $p$, a triple; $B$, a set of triples

Output: $\mathcal{L}$-normal form of $\operatorname{poly}(p)$ modulo $\operatorname{poly}(B)$

1: $h:=\operatorname{poly}(p)$;

2: $G:=\operatorname{poly}(B)$;

3: while $h$ has a term $t$ which is $\mathcal{L}$-reducible modulo $G$ do

4: $\quad$ Select $g \in G$ with $\left.\operatorname{LM}(g)\right|_{\mathcal{L} t}$;

5: $\quad h:=h-g \frac{t}{\mathrm{LT}(g)}$;

6: od

7: Return $(h)$ 
Subalgorithm HEADNORMALFORM

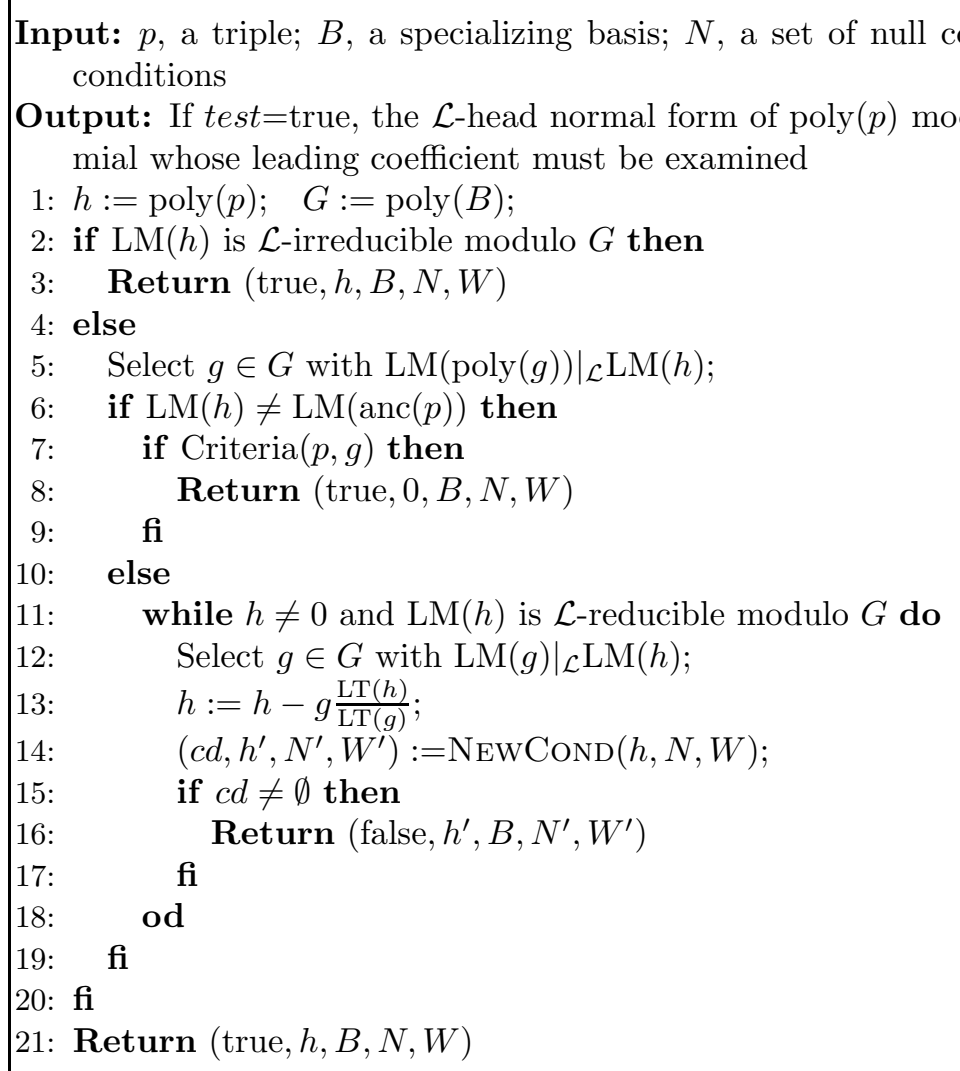

Theorem 2. Algorithm ComInvSYS terminates in finitely many steps, and computes a minimal CIS for its input ideal.

Proof. Let $I=\langle F\rangle$ where $F=\left\{f_{1}, \ldots, f_{k}\right\} \subset K[\mathbf{a}, \mathbf{x}]$ is a parametric set, $\mathbf{x}=x_{1}, \ldots, x_{n}$ (resp. $\left.\mathbf{a}=a_{1}, \ldots, a_{m}\right)$ is a sequence of variables (resp. parameters). Let $\prec_{\mathbf{x}}$ (resp. $\prec_{\mathbf{a}}$ ) be a monomial ordering involving the $x_{i}$ 's (resp. $a_{i}$ 's), and $\mathcal{L}$ be an involutive division on $K[\mathbf{x}]$.

Suppose that ComInvSYs receives $F$ as an input. To prove the termination, we use the fact that $K[\mathbf{a}]$ is a Noetherian ring. When BRANCH is called, the leading coefficient of some polynomial $f \in I$ is analyzed. For this purpose, the subalgorithm NewCond determines whether LC $(f)$ is decidable or not w.r.t. the given specification $(N, W)$. Two alternative cases can take place:

- $\operatorname{LC}(f)$ is decidable and we check the global variable ind. Now if ind $<k$, then we study the next polynomial in $F$. Otherwise, GBI is called. If all the leading coefficients of the examined polynomials (to compute a minimal involutive basis) are decidable, then the output, say $G$, is a minimal involutive basis of $I$ w.r.t. $(N, W)$, and we add $(G, N, W)$ to List. Otherwise, two new branches are created by calling BRANCH (cf. the second case given below). In doing so, the minimality of $G$ and the termination of its computation is provided by the structure of GBI algorithm (see [6]).

- $\mathrm{LC}(f)$ is not decidable and we create two branches with $(N, W \cup c d)$ and $(N \cup c d, W)$, where $c d$ is the one-element set containing the new condition derived from $\operatorname{LC}(f)$.

Thus, in the second case, the branch for which $N$ (resp. $W$ ) is assumed, increases the ideal $\langle N\rangle \subset K[\mathbf{a}]$ (resp. $\langle W\rangle \subset K[\mathbf{a}])$. Note that we replace $N$ by a Gröbner basis of its ideal (see line 4 in NewCond). Since the ascending chains of ideals stabilize, the algorithm terminates. This argument was inspired by the proof in [13], Theorem 16.

To prove the correctness, assume that $M=\left\{\left(G_{i}, N_{i}, W_{i}\right)\right\}_{i=1}^{\ell}$ is the output of ComInvSys for the input is $F$ (note that we have used the fact the this algorithm terminates in finitely many 
steps). Consider integer $1 \leq i \leq \ell$ homomorphism $\sigma: K[\mathbf{a}] \rightarrow K^{\prime}$ where $\left(N_{i}, W_{i}\right)$ is a specification of $\sigma$ and $K^{\prime}$ is a field extension of $K$.

We have to show that for each $f \in G_{i}$ and each $x \in N M_{\mathcal{L}}\left(\operatorname{LM}(\sigma(f)), \operatorname{LM}\left(\sigma\left(G_{i}\right)\right)\right)$, in accordance with Theorem 1 , the equality $\mathrm{NF}_{\mathcal{L}}\left(\sigma(x f), \sigma\left(G_{i}\right)\right)=0$ holds. By using 'reductio ad absurdum', suppose $g=\mathrm{NF}_{\mathcal{L}}\left(\sigma(x f), \sigma\left(G_{i}\right)\right)$ and $g \neq 0$. Since $\left(G_{i}, N_{i}, W_{i}\right)$ has been added to List in BRANCH, the leading coefficients of the polynomials in the subalgorithm GBI, examined at computation of a minimal involutive basis for $F$, are decidable w.r.t. $\left(N_{i}, W_{i}\right)$. Furthermore, $f \in G_{i}$ implies that in the course of GBI $x f$ is added to $Q$, the set of all nonmultiplicative prolongations that must be examined (see the notations used in GBI). Then, HEADREDUCE is called to perform the $\mathcal{L}$-head reduction of the elements of $Q$ modulo the last computed basis $T \subset G_{i}$. The computed $\mathcal{L}$-head normal form of $x f$ is further reduced by invoking TAILNORMALFORM which performs the $\mathcal{L}$-tail reduction. By the above notations, $g$ is the result of this step. Thus, $g$ should be added to $T \subset G_{i}$. It follows that $\mathrm{NF}_{\mathcal{L}}\left(\sigma(x f), \sigma\left(G_{i}\right)\right)=0$, a contradiction, and this completes the proof.

\section{Example}

Now we give an example to illustrate the step by step construction of a minimal CIS by the algorithm ComInvSys proposed and described in the previous section ${ }^{3}$.

For the input $F=\left\{a x^{2}, b y^{2}\right\} \subset \mathbb{K}[a, b, x, y]$ from Example 2, Janet division and the lexicographic monomial ordering with $b \prec_{\operatorname{lex}} a$ and $y \prec_{\operatorname{lex}} x$ the algorithm performs as follows:

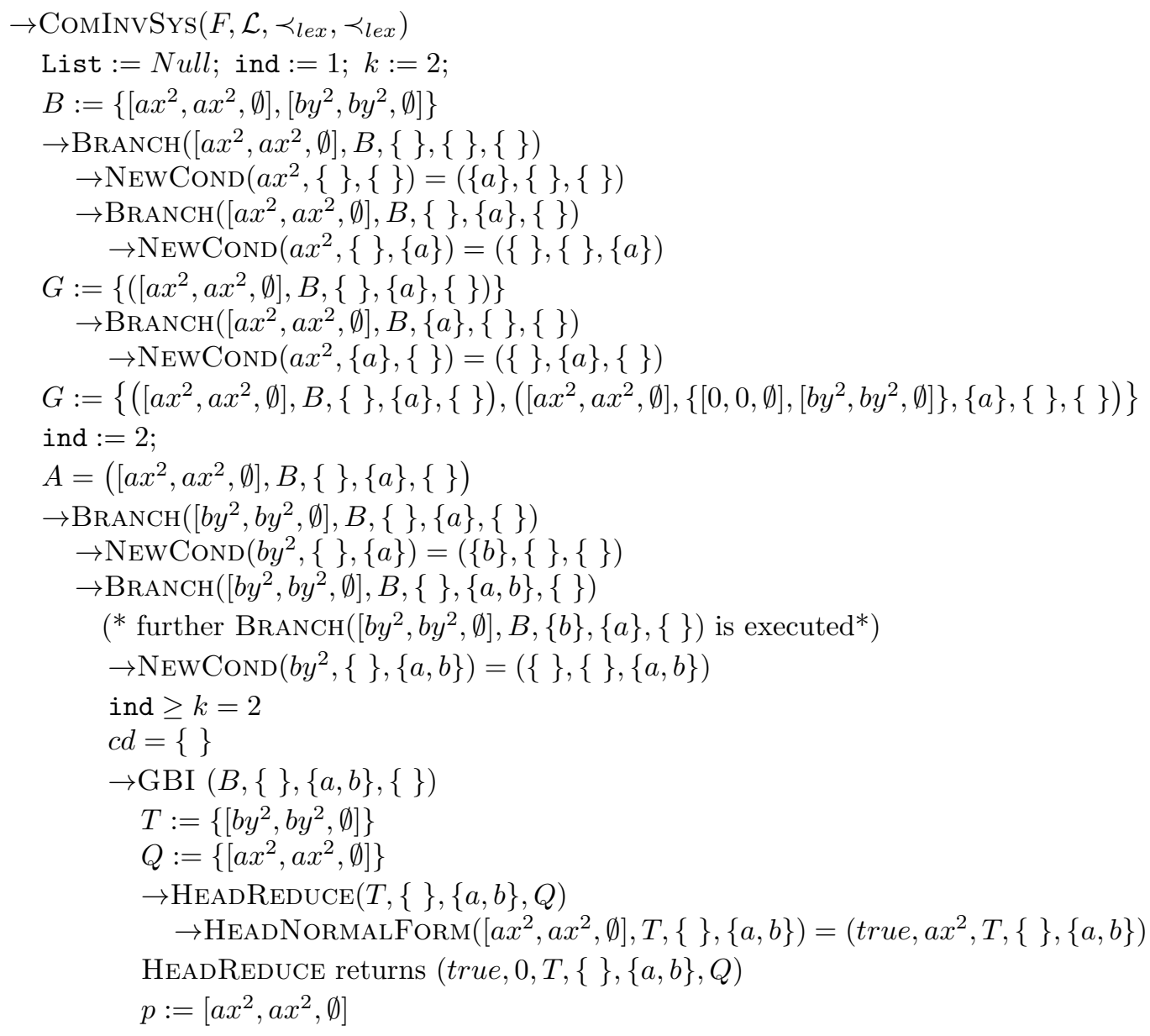

\footnotetext{
${ }^{3}$ The Maple code of our implementation of the algorithm for the Janet division [7] is available at http://invo.jinr.ru and http://amirhashemi.iut.ac.ir/software.html
} 


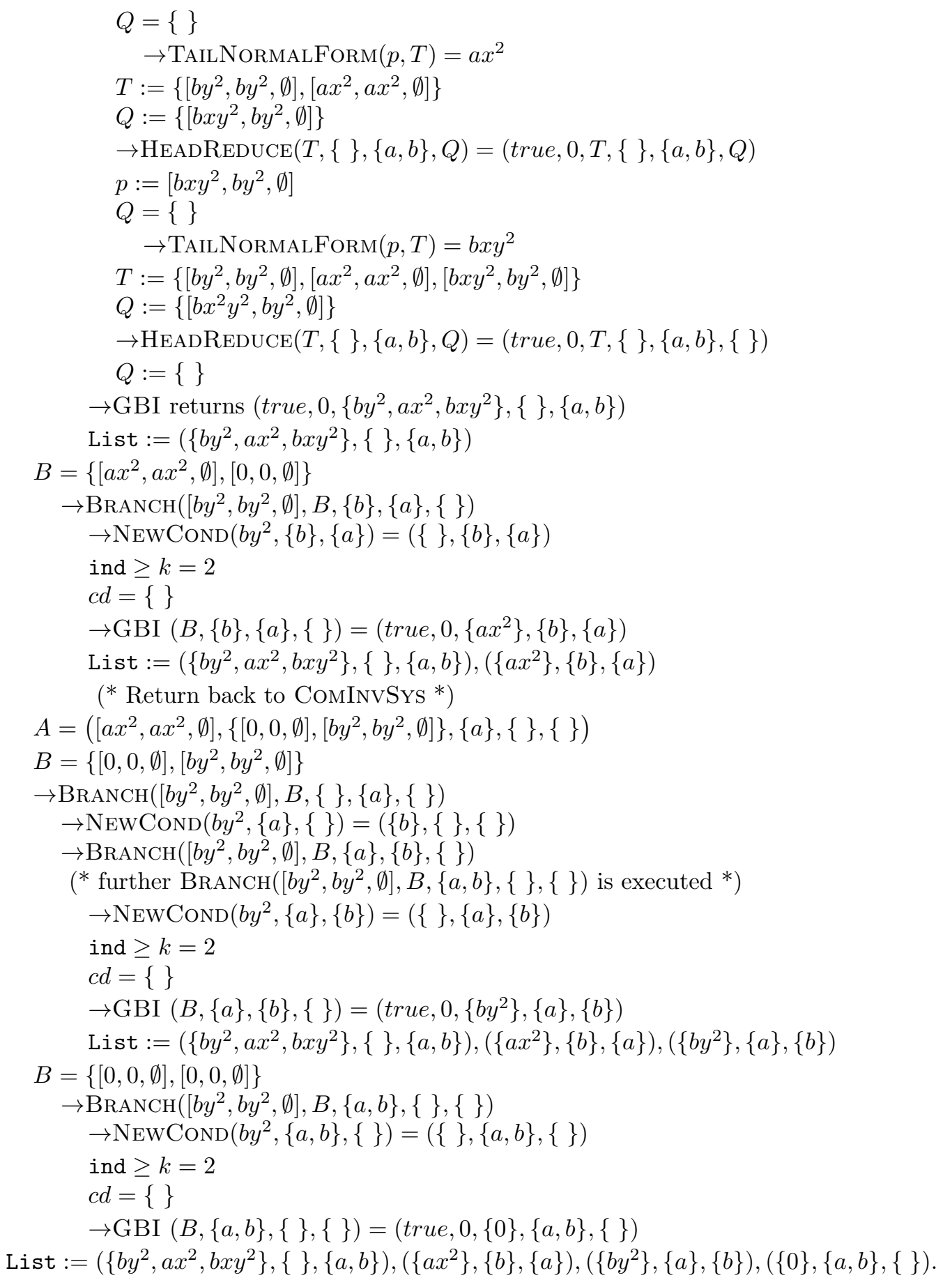

\section{Acknowledgements}

The main part of research presented in the paper was done during the stay of the second author (A.H.) at the Joint Institute for Nuclear Research in Dubna, Russia. He would like to thank the first author (V.G.) for the invitation, hospitality, and support. The contribution of the first author was partially supported by grants 01-01-00200, 12-07-00294 from the Russian Foundation for Basic Research and by grant 3802.2012.2 from the Ministry of Education and Science of the Russian Federation. 


\section{References}

1. Apel, J., Hemmecke, R.: Detecting unnecessary reductions in an involutive basis computation. J. Symbolic Computation 40, 1131-1149 (2005)

2. Becker, T., Weispfenning, T.: Gröbner Bases: a Computational Approach to Commutative Algebra. Graduate Texts in Mathematics, 141, Springer-Verlag, New York (1993)

3. Buchberger, B.: Ein Algorithms zum Auffinden der Basiselemente des Restklassenrings nach einem nuildimensionalen Polynomideal. PhD thesis, Universität Innsbruck (1965)

4. Buchberger, B.: A Criterion for Detecting Unnecessary Reductions in the Construction of Gröbner Bases. In: Edward W. Ng (ed.) EUROSAM'79. LNCS, vol.72, pp. 3-21. Springer, Berlin (1979)

5. Buchberger, B., Winkler, F. (eds.): Gröbner Bases and Applications. London Mathematical Society Lecture Note Series, Vol. 251. Cambridge University Press, Cambridge (1998)

6. Gerdt, V.P.: Involutive Algorithms for Computing Gröbner Bases. In: Cojocaru, S. Pfister, G., Ufnarovski, V. (eds.) Computational Commutative and Non-Commutative Algebraic Geometry, pp. 199225. IOS Press, Amstrerdam (2005) (arXiv:math/0501111)

7. Gerdt, V.P., Blinkov, Yu.A.: Involutive Bases of Polynomial Ideals. Mathematics and Computers in Simulation 45, 519-542 (1998)

8. Gerdt, V.P., Blinkov, Yu.A.: Involutive Division Generated by an Antigraded Monomial Ordering. In: Gerdt, V.P., Koepf, W., Mayr, E., Vorozhtsov, E.V. (eds.) CASC 2011. LNCS, vol. 6885, pp. $158-174$. Springer, Berlin (2011)

9. Janet, M.: Les Systèmes d'Équations aux Dérivées Partielles. Journal de Mathématique 3, 65-151 (1920)

10. Kapur, D., Sun, Y., Wand, D.: A New Algorithm for Computing Comprehensive Gröbner Systems. In: Watt, S.M. (ed.) Proc. ISSAC'10, pp. 29-36. ACM Press, New York (2010)

11. Manubens, M., Montes, A.: Improving DisPGB algorithm using the discriminant ideal. J. Symbolic Computation 41, 1245-1263 (2006)

12. Manubens, M., Montes, A.: Minimal Canonical Comprehensive Gröbner Systems. J. Symbolic Computation 44, 463-478 (2009)

13. Montes, A.: A new algorithm for discussing Gröbner bases with parameters. J. Symbolic Computation 33, 183-208 (2002)

14. Montes, A.: Solving the load flow problem using Gröbner bases. SIGSAM Bulletin 29, 1-13 (1995)

15. Montes, A., Wibmer, M.: Gröbner bases for polynomial systems with parameters. J. Symbolic Computation 45, 1391-1425 (2010)

16. Pommaret, J.-F.: Systems of Partial Differential Equations and Lie Pseudogroups. Mathematics and its Applications, vol. 14. Gordon \& Breach Science Publishers, New York (1978)

17. Sato, Y., Suzuki, A.: An alternative approach to comprehensive Gröbner bases. J. Symbolic Computation 36, 649-667 (2003)

18. Sato, Y., Suzuki, A.: A simple algorithm to compute comprehensive Gröbner bases using Gröbner bases. In: Trager, B.M. (ed.) Proc. ISSAC'2006, pp. 326-331. ACM Press, New York (2006)

19. Suzuki, A.: Computation of full comprehensive Gröbner bases. In: Ganzha, V.G, Mayr, E., Vorozhtsov, E.V. (eds.) CASC 2005. LNCS, vol. 3781, pp. 431-444. Springer, Berlin (2005)

20. Seiler, W.M.: Involution - The Formal Theory of Differential Equations and its Applications in Computer Algebra. Algorithms and Computation in Mathematics, vol. 24. Springer-Verlag, Berlin (2010)

21. Weispfenning, V.: Cannonical comprehensive Gröbner bases. J. Symbolic Computation 36, 669-683 (2003)

22. Weispfenning, V.: Comprehensive Gröbner Bases. J. Symbolic Computation 14, 1-29 (1992)

23. Zharkov, A.Yu., Blinkov, Yu.A.: Involutive approach to investigating polynomial systems. Mathematics and Computers in Simulation 42, 323-332 (1996) 